\title{
Antiemetic Effects of Granisetron or Metoclopramide Administered Prior to Amrubicin Monotherapy
}

\author{
Takeshi Aoyama ${ }^{1,2}$, Kenichi Suzuki ${ }^{1}$, Takashi Tsunoda ${ }^{1}$, Kazuyoshi Kawakami ${ }^{1}$, Tomomi Takiguchi ${ }^{1}$, \\ Hiroshi Watanabe ${ }^{1}$, Wataru Suzuki1 ${ }^{1}$ Kazuo Sugita ${ }^{1}$, Hitoshi Sato ${ }^{2}$, Erika Sugiyama², Toshihiro Hama ${ }^{1}$, and \\ Makoto Nishio ${ }^{3}$ \\ 1. Department of Pharmacy, The Cancer Institute Hospital, Japanese Foundation for Cancer Research, 3-8-31 Ariake, Koto-ku, Tokyo \\ 135-8550, Japan. \\ 2. Department of Drug Information, Division of Pharmacokinetics/Pharmacodynamics, School of Pharmacy, Showa University, Tokyo \\ 142-8555, Japan. \\ 3. Department of Thoracic Medical Oncology, The Cancer Institute Hospital, Japanese Foundation for Cancer Research, 3-8-31 Ariake, \\ Koto-ku, Tokyo 135-8550, Japan.
}

$\triangle$ Corresponding author: Takeshi Aoyama at the Japanese Foundation for Cancer Research, Cancer Institute Hospital, Department of Pharmacy, 3-8-31 Ariake Koto-ku, Tokyo 135-8550, Japan. Tel: +81-3-3570-0215(Direct); Fax: +81-3-3570-0216; E-mail: takeshi.aoyama@jfcr.or.jp.

(c) Ivyspring International Publisher. This is an open access article distributed under the terms of the Creative Commons Attribution (CC BY-NC) license (https://creativecommons.org/licenses/by-nc/4.0/). See http://ivyspring.com/terms for full terms and conditions.

Received: 2016.09.22; Accepted: 2016.10.10; Published: 2017.01.01

\begin{abstract}
Purpose: Amrubicin (AMR) is an anthracycline antitumor agent that has little cardiotoxicity and exhibits promising activity for small cell lung cancer (SCLC). Although AMR monotherapy is primarily used as a second-line treatment for SCLC in Japan, AMR-induced nausea and vomiting have not been definitively evaluated, and antiemetic therapy for AMR has yet to be established. This study retrospectively evaluated the antiemetic effects of granisetron or metoclopramide during AMR monotherapy.

Methods: We reviewed the medical records of SCLC patients who received AMR monotherapy at our hospital. Incidence of nausea and vomiting and the complete response (CR) of the anti-emesis, which was defined as the absence of any emetic episode or the use of any rescue medication, were evaluated.

Results: A total of 63 SCLC patients were treated with AMR monotherapy between April 2009 and November 2014. Antiemetic therapy was administered on days 1-3, with 33 patients receiving $3 \mathrm{mg}$ of granisetron (Group G) and 30 patients receiving $20 \mathrm{mg}$ of metoclopramide (Group M). Nausea and vomiting were observed in $30.3 \%$ of Group $G$ and $30.0 \%$ of Group M patients. The CR rate observed was $78.7 \%$ in Group $G$ and $73.3 \%$ in Group M. There were no significant differences between Groups $\mathrm{G}$ and $\mathrm{M}$.

Conclusion: Both granisetron and metoclopramide were successfully used for anti-emesis during AMR monotherapy.
\end{abstract}

Key words: Anti-emesis - Amrubicin monotherapy- Granisetron -Metoclopramide-Moderately emetic-risk chemotherapy.

\section{Introduction}

Amrubicin (AMR) is an anthracycline antitumor agent and potent topoisomerase II inhibitor [1]. In contrast to other anthracyclin agents, AMR has little cardiotoxicity $[2,3]$. After the approval of AMR in
Japan for small cell lung cancer (SCLC) and non-small-cell lung cancer (NSCLC), this drug has been evaluated in a number of Japanese studies for use as a second-line treatment for $\operatorname{SCLC}[4,5]$. The 
Japan Society of Clinical Oncology Guidelines for the Optimal Use of Antiemetics (the Japanese Guidelines) have classified the nausea and vomiting associated with AMR as a moderate emetic risk chemotherapy (MEC) [6]. Although $57.6 \%$ of patients experienced nausea and vomiting during the phase II clinical trial (WJTOG study) that was conducted in Japan, grade 3 or higher nausea and vomiting were not observed during this trial [7]. However, since the WJTOG study sample size was small, the prophylactic antiemetic therapy during the observational period could not be clarified. Moreover, it should be noted that prior to the publication of the Japanese guidelines, the Cancer Institute Hospital of the Japanese Foundation of Cancer Research has been using both granisetron and metoclopramide for AMR monotherapy. Thus, as the nausea and vomiting of AMR have not been definitively evaluated, the antiemetic effect for the AMR therapy has yet to be established.

Therefore, the aim of the current study was to retrospectively evaluate the antiemetic effect of granisetron and metoclopramide during AMR monotherapy.

\section{Methods}

\section{Patient selection}

We reviewed the medical records of SCLC patients who received AMR monotherapy between April 2009 and November 2014. After being admitted to our hospital, all of the patients received their first cycle of the AMR monotherapy, which was administered at the Thoracic Medical Oncology Department. All patients were hospitalized for at least 8 days after the initiation of their treatment. Patients were excluded from the study if they had had nausea and/or vomiting or had received following medication at the time of initiation of AMR monotherapy: dopamine antagonists, steroids, phenothiazines, or benzodiazepines.

\section{Study design and treatment regimen}

This retrospective observational study examined AMR monotherapy patients who underwent 3 sequential days of AMR administration with at least an 18-day interval between the cycles. Immediately prior to the AMR administration, patients received either $3 \mathrm{mg}$ of granisetron (Group G) or $20 \mathrm{mg}$ of metoclopramide (Group M) as prophylactic therapy for emesis. The ethics committees of our institutions approved the protocol for this study.

\section{Assessment}

We investigated patients' emetic episodes and use of antiemetic drugs during the first 8 days from their initial day of AMR monotherapy. Subsequently, we assessed the severity of their nausea and vomiting using the Common Terminology Criteria for Adverse Events (CTCAE) version 4.0, and derived the incidence of nausea and vomiting for each grade. In addition, we derived the complete response (CR) rate of anti-emesis which was defined as the absence of any emetic episode or the use of rescue medication for Groups $\mathrm{G}$ and $\mathrm{M}$ during the 8-day observational period.

\section{Statistical analysis}

Groups $\mathrm{G}$ and $\mathrm{M}$ were compared using the Mann-Whitney $U$ test, $\chi^{2}$ test and Fisher's exact test, as appropriate. $P$ values of less than 0.05 (two sided) were considered to indicate statistical significance. $\mathrm{R}$ version 3.0 ( $R$ Core Team (2013)) was used for all statistical analyses.

\section{Results}

Table 1 presents the patient characteristics. Out of the 63 total patients, 33 received $3 \mathrm{mg}$ of granisetron (Group G), while 30 received $20 \mathrm{mg}$ of metoclopramide (Group M) as antiemetic therapy.

No differences were observed between the groups for age, gender, ECOG performance status, opioid use, and chemotherapy dose. The study dose in the majority of the patients in both groups was 40 $\mathrm{mg} / \mathrm{m}^{2}$, with this dose used in $26(78.7 \%)$ of the patients in Group G and in $25(83.3 \%)$ of the patients in Group M.

Table 2 presents the incidence of nausea and vomiting during the 8 days after the treatment initiation. Grade 1 nausea was observed in 10 patients $(30.3 \%)$ of Group G, with 23 other patients $(69.6 \%)$ exhibiting no nausea episodes during the 8-day period.

Grade 1 nausea occurred in 7 patients $(23.3 \%)$ of Group M, while grade 2 nausea was observed in 2 patients $(6.6 \%)$. There were 21 other patients $(70.0 \%)$ in this group who did not exhibit any nausea episodes during the 8-day period.

During the 8-day observation period, there were no significant differences $(P>0.05)$ found for the occurrence of nausea between Group $\mathrm{G}$ and $\mathrm{M}$.

Grade 1 vomiting occurred in 2 patients $(6.0 \%)$ of Group G, while the other 31 patients $(93.9 \%)$ did not exhibit any vomiting episodes during the 8-day period. In Group M, grade 1 vomiting occurred in 1 patient (3.3\%), with the other 29 patients (96.6\%) exhibiting no vomiting episodes during the 8-day period.

During the 8-day observation period, there were no significant differences $(P>0.05)$ observed for the occurrence of vomiting between Group $\mathrm{G}$ and $\mathrm{M}$. 
Table 1. Baseline Demographic and Clinical Characteristics.

\begin{tabular}{|c|c|c|c|}
\hline & $\begin{array}{c}\text { Granisetron }(n=33) \\
n(\%)\end{array}$ & $\begin{array}{c}\text { Metoclopramide }(n=30) \\
n(\%)\end{array}$ & $P$ value \\
\hline \multicolumn{4}{|l|}{ Age, years } \\
\hline Median & 69 & 68 & 0.863 \\
\hline Range & $56-84$ & $52-81$ & \\
\hline \multicolumn{4}{|c|}{ Amrubicin dose $\left(\mathrm{mg} / \mathrm{m}^{2}\right)$} \\
\hline $30 / 35 / 40$ & 0 / 7 ( 21.2 ) / 26 (78.7) & $1(3.3) / 4(13.3) / 25(83.3)$ & 0.514 \\
\hline \multicolumn{4}{|l|}{ Sex, n (\%) } \\
\hline Male/ Female & 31 (93.9) / 2 (6.0) & $25(83.3) / 5(16.6)$ & 0.242 \\
\hline Opioid use history & $4(12.1)$ & $3(10.0)$ & 0.853 \\
\hline \multicolumn{4}{|c|}{ ECOG performance status } \\
\hline $0 / 1 / 2 / 3 /$ & $16(48.4) / 15(45.4) / 1(3.0) / 1(3.0)$ & $16(53.3) / 11(36.6) / 2(6.6) / 1(3.3)$ & 0.846 \\
\hline
\end{tabular}

Table 2. Incidence of nausea and vomiting.

\begin{tabular}{lccccccccccc}
\hline & \multicolumn{4}{c}{ Nausea n (\%) } & \multicolumn{3}{c}{ Vomiting n (\%) } \\
\cline { 2 - 10 } & Grade 1 & Grade 2 & Grade 3 & Grade 4 & $\boldsymbol{P}$ value & Grade 1 & Grade 2 & Grade 3 & Grade 4 & $\boldsymbol{P}$ value \\
\hline Granisetron $\quad(\mathrm{n}=33)$ & $10(30.3)$ & 0 & 0 & 0 & 0.404 & $2(6.0)$ & 0 & 0 & 0 & 0.999 \\
Metoclopramide $(\mathrm{n}=30)$ & $7(23.3)$ & $2(6.6)$ & 0 & 0 & & $1(3.3)$ & 0 & 0 & 0 & 0 \\
\hline
\end{tabular}

Incidence of nausea and vomiting according to grade. Statistical tests were performed using a $\chi^{2}$ test (two sided).

Figure 1 presents the $C R$ rates that were observed during the 8-day period. The $\mathrm{CR}$ rate in Group G was $78.7 \%$, with 7 of the patients given rescue medication after they did not achieve $C R$ within the 8 days. The CR rate in Group $M$ was $73.3 \%$, with 8 patients given rescue medication after they did not achieve $C R$ within the 8 days.

During the 8-day observation period, there were no significant differences $(P>0.05)$ found for the $C R$ between Group $\mathrm{G}$ and M.

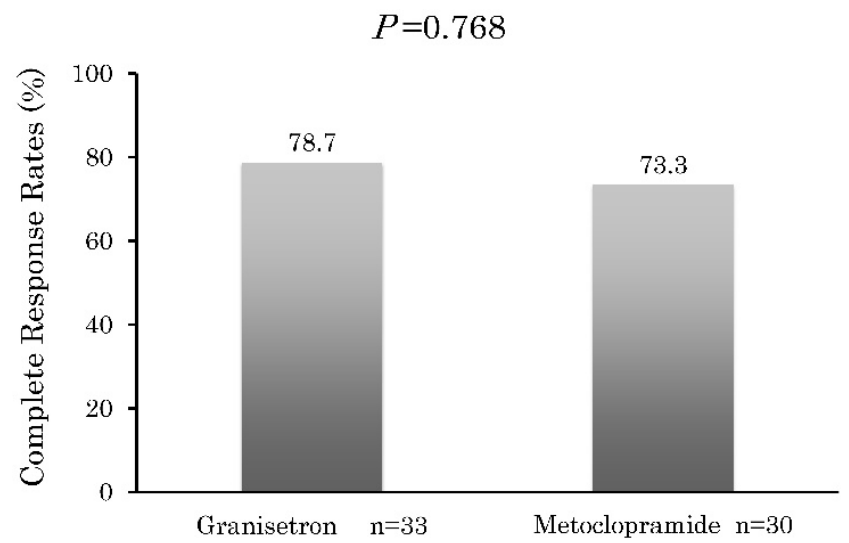

Fig. 1 Complete response. Percentage of patients with a complete response after treatment with granisetron or metoclopramide in addition to having no emetic episodes or any receiving any rescue medication. Statistical tests were performed using a $\mathrm{X} 2$ test (two sided).

\section{Discussion}

The results for this study demonstrated that there were no significant differences for either the incidence of nausea and vomiting or for the CR rate between Groups G and M. The current Japanese Guidelines state that AMR possesses a moderate risk of chemotherapy-induced nausea and vomiting (CINV), and thus they recommend that dexamethasone and 5-hydroxytryptamine-3 receptor antagonist (5- $\left.\mathrm{HT}_{3} \mathrm{RA}\right)$ be used for antiemetic therapy. In contrast to the recommendations of the guidelines, the current study found that AMR-induced nausea and vomiting were well controlled by either $3 \mathrm{mg}$ of granisetron or $20 \mathrm{mg}$ of metoclopramide.

We believe there may be two reasons for our current results. First, as per the anti-emesis guidelines, the risk of CINV is determined by the incidence of nausea and vomiting that occurs within 24 hours after the initial administration without any prior prophylactic antiemetic therapy. During the WJTOG study, which served as the basis of the Japanese Guidelines, AMR-induced nausea and vomiting were observed in $57.6 \%$ of the patients. However, both the observational period and the prophylactic therapy used were not clearly defined, and there was only a small sample size. Since this has been the only study that has specifically tried to evaluate these 
occurrences, the accuracy of the incidence of CINV remains in doubt.

The second reason that may explain our results involves the AMR dose. During the WJTOG study, the designated AMR dose was set at $45 \mathrm{mg} / \mathrm{m}^{2}$. However, due to the intolerability for myelosuppression, 40 $\mathrm{mg} / \mathrm{m}^{2}$ has been the consensus for current practical use. Thus, in our current study, all of the doses used were $40 \mathrm{mg} / \mathrm{m}^{2}$ or less.

Furthermore, AMR-induced nausea and vomiting were observed in $30.3 \%$ of the Group G patients and in $30.0 \%$ of the Group $M$ patients. In addition, there were only 7 patients $(21.2 \%)$ in Group $\mathrm{G}$ and 8 patients $(26.6 \%)$ in Group $\mathrm{M}$ who were not able to achieve CR. These results suggest that the emetogenicity of AMR is lower than the MEC designation by the Japanese Guidelines. Considering the above, dexamethasone alone appears to be a feasible option because it is recommended by the guidelines as an antiemetic therapy during low emetic-risk chemotherapy (LEC). However, randomized controlled trials of antiemetic therapy for antitumor agents that have a low risk of acute vomiting have yet to be undertaken. Therefore, we believe that granisetron or metoclopramide are possible options for AMR monotherapy.

Metoclopramide is one of the most frequently used dopamine antagonists. In Japan, this drug is considered to be one of the options when an antiemetic therapy with LEC is required. When high doses of metoclopramide are used in order to prevent CINV, extrapyramidal symptoms (EPS) have been reported to emerge [8,9]. However, only one patient in each report experienced EPS. In addition, there is also a report that says no EPS were observed in their study patients, who were administered high doses of metoclopramide for the purpose of preventing CINV [10].On the other hand, previous meta-analyses have shown that $10 \mathrm{mg}$ of metoclopramide was not effective when used for postoperative nausea and vomiting [11]. However, when the dose was increased to $20 \mathrm{mg}$ of metoclopramide, the drug proved to have similar effect for nausea and vomiting of postoperative patients to ondansetron $8 \mathrm{mg}$ [12].

These results indicated that a $20 \mathrm{mg}$ low dose of metoclopramide might be an appropriate antiemetic therapy for AMR monotherapy.

From an economic standpoint, the cost of metoclopramide compared with a 5- $\mathrm{HT}_{3} \mathrm{RA}$ antagonist is lower. For example, in Japan, the cost of $3 \mathrm{mg}$ of granisetron is approximately 42 times greater than the cost of $20 \mathrm{mg}$ of metoclopramide. Therefore, metoclopramide is beneficial from a pharmacoeconomics standpoint.
One limitation of our current study is that this was not a prospective randomized controlled study. Furthermore, there were no interventions with regard to the treatments. Thus, it is possible that there could have been some bias in our results. Future investigations that examine the efficacy of $20 \mathrm{mg}$ of metoclopramide in clinical situations that involve low risk chemotherapy will need to be undertaken.

In conclusion, our results indicated that both granisetron and metoclopramide are feasible options for use as anti-emesis during AMR monotherapy.

\section{Competing Interests}

The authors have declared that no competing interest exists.

\section{References}

1. Hanada M, Mizuno S, Fukushima A, et al. A new antitumor agent amrubicin induces cell growth inhibition by stabilizing topoisomerase II-DNA complex. Jpn J Cancer Res. 1998; 89: 1229-38.

2. Suzuki T, Minamide S, Iwasaki T, et al. Cardiotoxicity of a new anthracycline derivative (SM-5887) following intravenous administration to rabbits: comparative study with doxorubicin. Invest New Drugs. 1997; 15: 219-225.

3. Noda T, Watanabe T, Kohda A, et al. T. Chronic effects of a novel synthetic anthracycline derivative (SM-5887) on normal heart and doxorubicin-induced cardiomyopathy in beagle dogs. Invest New Drugs. 1998; 16: 121-8.

4. Inoue A, Sugawara S, Yamazaki K, et al. Randomized phase II trial comparing amrubicin with topotecan in patients with previously treated small-cell lung cancer: North Japan Lung Cancer Study Group Trial 0402. J Clin Oncol. 2008; 20: 5401-6.

5. Onoda S, Masuda N, Seto T, et al. Phase II trial of amrubicin for treatment of refractory or relapsed small-cell lung cancer-Thoracic Oncology Research Group Study 0301. J Clin Oncol. 2006; 24: 5448- 5453.

6. Horie Y, Boku N. A guideline for antimetics use for patients with malignancies. Gan To Kagaku Ryoho. 2012; 39: 1163-1168.

7. Yana T, Negoro S, Takada M, et al. Phase II study of amrubicin in previously untreated patients with extensive-disease small cell lung cancer: West Japan Thoracic Oncology Group (WJTOG) study. Invest New Drugs. 2007; 25: 253-258.

8. Gralla RJ, Itri LM, Pisko SE. et al. Antiemetic efficacy of high-dose metoclopramide: randomized trials with placebo and prochlorperazine in patients with chemotherapy-induced nausea and vomiting. N Engl J Med. 1981; 305: 905-909.

9. Ithimakin S, Runglodvatana K, Nimmannit A et al. Randomized, double-blinded, placebo-controlled trial of ondansetron plus dexamethasone with or without metoclopramide as antiemetic prophylaxis in patients receiving high-dose cisplatin in medical practice. Support Care Cancer. 2012; 20: 849-855

10. Roila F, Ruggeri B, Ballatori E et al. Aprepitant versus metoclopramide, both combined with dexamethasone, for the prevention of cisplatin-induced delayed emesis: a randomized, double-blind study. Ann Oncol. 2015; 26: 1248-53.

11. Henzi I, Walder B, Tramer M.R. Metoclopramide in the prevention of postoperative nausea and vomiting: a quantitative systematic review of randomized, placebo-controlled studies. Br J Anaesth. 1999; 83: 761-771.

12. Quaynor H, Raeder JC. Incidence and severity of postoperative nausea and vomiting are similar after metoclopramide $20 \mathrm{mg}$ and ondansetron 8 $\mathrm{mg}$ given by the end of laparoscopic cholecystectomies. Acta Anaesthesiol Scand. 2002; 46: 109-113. 